\title{
Review
}

\section{Methadone: Does Stigma Play a Role as a Barrier to Treatment of Chronic Pain?}

\author{
Shalini Shah, MD and Sudhir Diwan, MD
}

From: Weil-Cornell Medical Center, New York, NY.

Dr. Shah is with the Department of Anesthesiology, Weil-Cornell Medical Center, New York, NY.

Dr. Diwan is Medical Director of the Division of Pain Medicine in the Department of Anesthesiology, and Director of the Tri-Institutional Pain Fellowship Program with Weil-Cornell Medical Center, New York, NY.

Address correspondence: Shalini Shah, MD

Department of Anesthesiology 525 E 68th St Box 124 Room M 305 New York, NY 10021

E-mail: shs9o96@nyp.org

Disclaimer: There was no external funding in the preparation of this manuscript.

Conflict of interest: None.

Manuscript received: 01/19/2010 Revised manuscript received: 03/04/2010

Accepted for publication: $03 / 22 / 2010$

Free full manuscript: www.painphysicianjournal.com
Introduction: The synthetic opioid methadone is a promising analgesic for the management of chronic neuropathic pain. Methadone therapy is increasing as its advantages are being realized over other opioids. Methadone's lack of known active metabolites, high oral bioavailability, low cost, and its additional receptor activity as an antagonist of $\mathrm{N}$-methyl-D-aspartate receptors make it an attractive analgesic.

Methods: We surveyed 550 pain physicians to determine their prescribing practices of methadone. The study was approved by our Institutional Review Board. A list of 550 pain physicians, which included practitioners in private practice, university settings, and community hospitals, were obtained and surveys sent via mail. The list was obtained through the American Pain Society's membership list. Out of 550 surveys sent, 124 replies were returned.

Results: The 124 surveys that were returned included pain physicians from various settings: 20 responses from physicians practicing at a university setting, 16 responses from a community setting, 54 responses from a private setting, one from university and community settings, 7 from community and private settings, 3 from university and community and private settings; 23 did not specify. Of the 124 physicians, 111 prescribe methadone in their pain practice. Of the 13 physicians who do not prescribe methadone, the main reason for not using the drug for 5 physicians was because of social stigma, 2 because of minimal experience with the drug, 2 because the drug was not effective, one because of lack of knowledge, and one because of potential adverse effects. Of the 111 physicians who use methadone, 55 stated that social stigma was the most common reason patients refuse to take methadone for the treatment of pain, 44 because of adverse effects, and 5 stated "other" as the reason patients refuse to take methadone. Of 111 physicians who prescribe methadone, 100 prescribed it for neuropathic pain, 101 for somatic pain, 80 for visceral pain, 78 for cancer pain, and 34 for sickle cell pain. Also, 21 stated that methadone was the primary opioid they prescribed. Of the 111 physicians who prescribe methadone, 86 start methadone at low dose and titrate up to minimize side effects. Fourteen clinicians load methadone and titrate down to minimize adverse effects while maintaining analgesia.

Conclusion: The majority of survey responders (90\%) prescribed methadone in their pain practice, but on a very limited basis; $59 \%$ state $<20 \%$ of their patients are on methadone. Three times a day dosing schedule was the most typical regimen (57\%) while $77 \%$ prefer to titrate up on the dosage. It seems interesting that many clinicians do not prescribe methadone as a primary analgesic. One reason for this is due to the social stigma of its use in treatment of heroin addicts. Also, a lack of widely recognized treatment algorithms or guidelines to assist clinicians with opioid conversions and maintenance might be playing a role. The role of stigma as a barrier to adequate treatment of chronic pain among pain physicians prescribing practices is a fundamental, yet unexplored issue.

Key words: Methadone, chronic pain, behavior, stigma, survey, opioid, education, society

Pain Physician 2010; 13:289-293 
A pproximately $30 \%$ of the US population suffers from chronic pain (1). Effectively managing chronic pain is often challenging, oftentimes requiring a comprehensive approach in long-term multidisciplinary pain centers. This approach includes multimodal treatment, both pharmacologic and nonpharmacologic interventions, physical therapy programs, and interventional treatment modalities. The success of these treatments is further compounded with the patient's support systems and education both in society and of their pain care providers. Social interactions, whether with professionals or family or friends, are important since they help to shape the expectations, perceived needs, and responses of those attending pain centers (2). The role of stigma in chronic pain management is a fundamental yet unexplored issue as a barrier to adequate chronic pain treatment. The societal stigmatization of the patient as a "chronic pain" patient distances the patient from seeking adequate treatment; on the other end of the spectrum it can be physician prescribing practices that can limit appropriate treatment. Patients with chronic pain, without clear structured pathology, are stigmatized compared with patients who have clear, identifiable, structural pathology. Chronic pain patients on oral opioid medications can be further stigmatized by the public compared to interventional therapies. The perspective further changes when opioid therapy is specifically switched to methadone. Each of these factors - sociological, pharmacological, and professional-will be discussed as each is vital to the stigma of chronic pain. The aims of this study are to identify these stigmas, specifically those related to chronic pain of unclear etiology, opioid medications, and methadone, and how these stigmas may play a role in patient care, societal implications, physician prescribing practices, and as a barrier to treatment.

\section{Opioid Therapy for Chronic Pain Patients}

For millennia, opioids have been used to treat pain and are undeniably the most commonly prescribed medications for pain. It is estimated that $90 \%$ of patients presenting to pain centers and receiving treatment in such facilities are on opioids (3). Although still controversial, opioid use has also been steadily increasing in the last 2 decades in patients with noncancer pain $(4,5)$. Compared to the large population of pain patients receiving opiate therapy, and the vast spectrum of pain disorders necessitating such therapy, the options and variety of strong opioids available for oral use is limited. Included in the list of the most prescribed and acceptable opiates are morphine, oxycodone, hydromorphone, hydrocodone, oxymorphone, codeine, fentanyl, and methadone.

Opioids for chronic pain are considered the "gold standard" pain medication. They are well absorbed, generally well tolerated, and available in all forms for routes of delivery. Opioids can also be mixed with local anesthetics if used epidurally or intrathecally, further compounding their maximal pain benefit. However, significant limitations do exist, including the inability to treat neuropathic pain with opioids unless given in high doses (i.e. a right shift of the dose-response curve of opioids to neuropathic pain), and unclear long-term treatment plans (6). Furthermore, the question arises how often stable patients should follow up, and how to continue prescriptions when one physician leaves the group.

Historically, pain physicians have withheld from prescribing chronic opioids except for the treatment of cancer pain. While opioids are generally well tolerated in a majority of patients, there are inherent risks, such as the risk of possible addiction, dependence, mental cloudiness, decreased functioning, and variable responses to escalating doses. Moreover, physicians may have been reluctant to prescribe opioids and other controlled substances because of potential legal consequences secondary to drug abuse and/or diversion (7). Despite these potential risks, certain patients with chronic noncancer pain who have shown minimal benefit from other classes of medication are considered for chronic opioid therapy.

In a comprehensive 2008 study by Trescot et al (3) to evaluate the effectiveness of opioids in the treatment of chronic noncancer pain, it was concluded that for long term opioid therapy of 6 months or longer with improvement in function and reduction in pain, there is weak evidence for morphine and transdermal fentanyl. Furthermore, there is limited or a lack of evidence for all other controlled substances, including the most prescribed drugs, oxycodone and hydrocodone (3). The results of this review demonstrate that many patients were dissatisfied with adverse events or insufficient pain relief from commonly prescribed opioids.

\section{Methadone for Chronic Pain}

Methadone was developed in Germany in the late 1930s in anticipation of possible shortages of raw opium during the upcoming war (7). The drug was 
given the trade name Dolophine from the Latin "dolor" meaning pain and "fin" meaning end. Methadone was introduced into the United States in 1947 by Eli Lilly and Company as an analgesic. Since then, it has been best known for its use in treating narcotic addiction. A great deal of anecdotal evidence was available that methadone might prove effective in treating heroin withdrawal; it had even been used in some hospitals. For the first time, work produced at Rockefeller University in 1964 by Professor Vincent Dole, his wife Dr. Marie Nyswander, and colleague Dr. Mary Jeanne Kreek, officially studied and recognized methadone as a potential substitution therapy for illicit narcotic use (7). Their studies introduced a sweeping change in the notion that drug addiction was not necessarily a simple character flaw, but rather a disorder to be treated in the same way as other diseases. To date, methadone maintenance therapy has been the most systematically studied and successful pharmacotherapy for the treatment of drug addiction patients.

Methadone, a diphenylhetane, is a racemic mixture of $d$ - and I- enantiomers. Analgesic properties are owing to the I-isomer; I-methadone is 50 times more potent on opioid receptors than d-methadone. Consequently, I-methadone is also responsible for adverse effects similar to other opiates such as respiratory depression and sedation. Methadone is also an N-methyl-D-aspartate (NMDA) receptor antagonist that blocks morphine tolerance development and NMDA induced hyperalgesia (8). Methadone is unique among other potent opiates. Methadone's half life (t1/2) is approximately 72 hours. This affects dose changes and has important therapeutic consequences regarding possible accumulation. Its oral bioavailability is $50 \%$ greater than morphine. Peak concentration occurs within 2 hours, with duration of action extending from 4 to 8 hours from administration compared with morphine's 3-4 hours duration of action. The compound is lipophilic and highly protein bound, can easily cross placental barriers, and is excreted in breast milk. Methadone, like other oral opioids, is well absorbed from the gastrointestinal tract; however, unlike other opiates, metabolism via the CP450 system converts methadone to inactive metabolites pyrroline and pyrrolidine. Furthermore, systemically administered $d$ methadone prevents induced morphine tolerance, and at the same dose which it inhibits morphine tolerance, blocks NMDA hyperalgesia by virtue of its NMDA activity (9). Due to its unique anti-NMDA properties, methadone is the treatment of choice for opioid induced hyperalgesia, adding to its benefits for neuropathic pain.
Adverse effects which may limit its use are usually dose dependent. As an opioid, methadone is also subject to physical dependence, addiction, and tolerance which are not different from other opioids. However, addictive properties are less than morphine; tolerance is minimal while respiratory depression and physical dependence are the same as morphine. Respiratory depression and excessive sedation typically occur with escalating doses only and the adverse effects are more pronounced on initiation of therapy rather than during stable, steady state doses.

The most significant risk of parenteral administration of methadone, which is unique to this medication, is QT prolongation, which can lead to potentially fatal arrhythmias. In the case of methadone, as its use can be life saving, absolute QTC is not a contraindication for the use of IV methadone, but should be monitored closely (10). A more than $10 \%$ increase should prompt concern about torsades de pointes. Close monitoring is especially needed in the presence of other risk factors such as electrolyte abnormality, structural cardiac disease, or some congenital diseases. Its lack of known active metabolites, long duration, high bioavailability, low cost and its additional receptor activity as an antagonist of N-methyl-D-aspartate receptors (NMDA), makes methadone a promising analgesic for the management of chronic neuropathic pain. In a systematic review evaluating the effectiveness of opioids for non cancer pain, a study by Sandoval et al (11) reviewed oral methadone for chronic noncancer pain in over 21 trials inclusive of over 545 patients in which the indication for methadone was primarily ineffectiveness with previous treatments $(3,11)$. Five studies with 234 patients who had more than 6 months of follow-up were included; of these, meaningful improvement was seen in 154 patients indicating $66 \%$ response. Sandoval et al's (11) review showed that in all 21 studies, of the 526 patients included, 308 patients, or $59 \%$, responded with meaningful relief, with up to $50 \%$ citing adverse effects (the most common was nausea or vomiting) $(3,11)$. A study by Portenoy et al (12), which over a 3-year period, looked at sustained release oxycodone, found that $70-80 \%$ of patients stated pain was the same or improved with oxycodone, yet adverse effects were seen in $88 \%$. Methadone's low tolerance rate, high potency, and low adverse effect profile is favorable in compared with other strong oral opiates. Despite the pharmacological attractiveness of this drug, its association with addiction and known use among drug addicts might, however, be a barrier to its use and treatment. 


\section{Chains of Stigma}

Despite all of the progress that has been made in diagnostic procedures and the increasing number of treatment facilities available, the number of people suffering from chronic pain conditions seems to be growing constantly in all industrialized countries, a fact which is demonstrated impressively by the epidemiology of low back pain (13). "Chronic"meaning "life-determining," represents a functional and emotional turning point in the patient's life as one is diagnosed with such a disease as "chronic pain." Furthermore, chronic pain becomes a destructive stigma when society reduces the afflicted to the status of the chronically ill, as opposed to other well known long-term systemic conditions such as hypertension or diabetes, which do not carry any stereotype despite their chronicity. This is in part because the patient meets a sympathetic audience as he deals with these other lifelong chronic diseases.

"Stigma" was documented by Goffman (14) in the 1960s and has since been widely applied to groups suffering from a variety of physical and mental conditions including chronic illness $(14,15)$. Since chronic pain is a subjective presentation, it is not in itself a visible entity in the human body; its manifestations in help-seeking behavior and treatment speak for its existence. However, as many previous authors have noted, it is this very invisibility of pain that allows society to discredit the sufferers (16). The stigmatization of those with chronic pain is evident in twentieth century literature which sought to identify the "pain-prone personality" (17), and attribute pain complaints to secondary gain (18). As a result, those diagnosed with chronic pain are misjudged by the public, and misdiagnosed by medical professionals and labeled with psychological issues. As such, these patients are labeled as complainers and are alienated from society. This leads them to find ways to hide their suffering. This forced need to conceal their disease is evidence of stigma. Consequently, stigmatization from the medical provider to the patient's condition often leaves the patient frustrated and confused by mixed messages and that might create a major barrier to successful treatment. Therefore, patient and family education is usually necessary when using opioids, including methadone for chronic pain.

How does social stigma exist in the treatment of chronic pain? Recent reports indicate that help-seeking from professionals for chronic pain is associated with substantial discrimination and prejudice from the general public and their pain providers (19). As such, these patients are more likely to have their pain under treated (20), which may be related to several factors: the patients' difficulty in identifying or describing their pain; physicians' concern about addiction, abuse, aberrant behavior and possible future withdrawal symptoms with opioid cessation; and misinformation about opioid pharmacology and tolerance $(21,22)$. Wessley et al (23) observed: "If you have to prove you are ill, you can't get well," a phenomenon referred to by some authors as "social iatrogenesis" $(2,23,24)$. Similar findings led Harding et al (25) to assert that "loss of trust in the medical profession needs to be addressed during the treatment of chronic pain, or poor outcomes and patient dissatisfaction are likely to continue."

Stigma also exists on the part of pain physicians in making pharmacologic choices to treat chronic pain. Certainly, prescribing habits can be a serious barrier to overcome. With today's diagnostic precision, failure to pinpoint an etiology of chronic pain would generally be seen as a lack of validation of symptoms (26). Added to this, frustration with commonly prescribed and generally effective medications cause physicians to be reluctant to prescribe methadone as a first line medication from more popular brand name opioids such as OxyContin. The stigma is further heightened when opioids are switched to methadone over brand name opioids, suggesting the possibility of abuse potential or diversion on an otherwise compliant patient. Lack of depth of education, compounded with patients with a history of chemical dependency or lack of comfort level with methadone makes the pain physician hesitant to endorse the medication. Moreover, physicians do not seem to be able to effectively educate the patient as to why methadone is an appropriate drug of choice, nor convince patients that the drug has elevated itself as a dual functioning agent, and oftentimes, is best in its class for the type of pain being experienced. In this era of rapid information and better understanding of receptor pharmacology, the stigma of methadone should not be a barrier to treatment of chronic pain.

\section{Conclusion}

It is apparent that stigma can be a substantial problem for people with chronic pain and may inhibit helpseeking from both informal and professional sources (19). Social stigma, compounded by prescriber stigma, severely limits appropriate medical care. There clearly is a need to address the stigma. This might involve both changing beliefs of the general public and challenging the perceptions of people with chronic pain. Further education for physicians by expert specialists is needed 
to change prescribing habits and increase comfort levels with the drug. Only then can education be passed onto patients and families and ultimately society into the development of constructive interventions for chronic pain.

The chain of stigma exists and increases with respect to the treatment modality selected. Patients' treatment with oral opioids can be stigmatized due to potential opioid- related issues, such as physical dependence, addiction and tolerance compared with patients receiving interventional procedures. The most socially stigmatized patients are those treated with chronic methadone. Methadone is an excellent drug, as effective as other oral opioids, if not better for the reduction and improvement of chronic pain. Numerous studies have shown it is better for neuropathic pain, and is the treatment of choice for chemical hyperalgesia, which can be caused by long term use of other opioids. Unfortunately, the lack of depth of knowledge by pain care providers about the benefits of the medication and societal stigma still play a role as a barrier to treatment.

\section{References}

1. Moulin DE, Clark AJ, Speechley M, Morley-Forster PK. Chronic pain in Canada - prevalence, treatment, impact and the role of opioid analgesia. Pain Res Manag 2002; 7:179-184.

2. Holloway I, Sofaer-Bennett B, Walker J. The stigmatisation of people with chronic back pain. Disabil Rehabil 2007; 29:1456-1464.

3. Trescot AM, Glaser SE, Hansen $\mathrm{H}$, Benyamin R, Glaser SE, Adlaka R, Patel S, Manchikanti L. Effectiveness of opioids in the treatment of chronic non-cancer pain. Pain Physician 2008; 11:S181S200.

4. Raja SN, Haythornthwaite JA, Pappagallo M, Clark MR, Travison TG, Sabeen S, Royall RM, Max MB. Opioids versus antidepressants in postherpetic neuralgia: A randomized, placebo-controlled trial. Neurology 2002; 59:10151021.

5. Lamb L, Pereira JX, Shir Y. Nurse case management program of chronic pain patients treated with methadone. Pain Manag Nurs 2007; 8:130-138.

6. Dworkin RH, O’Connor AB, Backonja M, Farrar JT, Finnerup NP, Jensen TS, Kalso EA, Loeser JD, Miaskowski C, Nurmikko TJ, Portenoy RK, Rice AS, Stacey BR, Treed RD, Turk DC, Wallace MS. Pharmacologic management of neuropathic pain: evidence- based recommendations. Pain 2007; 132:237-251.

7. Angel J. Anesthesia Secrets. 2nd ed. Appleton \& Lange, Norwalk, CT: 1996.

8. Lubenow T, Ivankovich A, Barkin R. Clinical Anesthesia. 5th ed. Lippincott Williams \& Wilkins, Philadelphia, 2006.
9. Davis A, Inturrisi C. D-Methadone blocks morphine tolerance and $\mathrm{N}$-methyl-d-aspartate-Induced hyperalgesia. J Pharm and Experimental Therapeutics (JPET) 1999; 289:1048-1053.

10. Shaiova L, Berger A, Blinderman C, Bruera $\mathrm{E}$, Davis MP, Derby S, Interussi C, Kallman J, Mehta D, Pappagallo M, Perlov, E. Consensus guideline on parenteral methadone use in pain and palliative care. Palliative and Supportive Care 2008; 6:165-176.

11. Sandoval JA, Furlan AD, Mailis-Gagnon A. Oral methadone for chronic noncancer pain: A systematic literature review of reasons for administration, prescription patterns, effectiveness, and side effects. Clin J Pain 2005; 21:503-512.

12. Portenoy RK, Farrar JT, Backonja MM, Cleeland CS, Yang K, Friedman M, Colucci SV, Richards P. Long-term use of controlled-release oxycodone for noncancer pain: Results of a 3-year registry study. Clin J Pain 2007; 23:287-299.

13. Muller-Busch HC. The "healthy pain patient”. Schmerz 1990; 4:179-183.

14. Goffman E. Social factors and disorders of communication. Mental symptoms and public order. Res Publ Assoc Res Nerv Ment Dis 1964; 42:262-9.

15. Charmaz K. Loss of self: A fundamental form of suffering in the chronically ill. Sociol Health Illn 1983; 5:168-195.

16. Glenton C. Chronic back pain sufferers - striving for the sick role. Soc Sci Med 2003; 57:2243-2252.

17. Engel GL. Psychogenic pain and painprone patient. Am J Med 1959; 26:899918.
18. Fishbain DA, Rosomoff HL, Cutler RB, Rosomoff RS. Secondary gain concept: A review of the scientific evidence. Clin J Pain 1995; 11:6-21.

19. Barney LJ, Griffiths KM, Christensen H, Jorm AF. Exploring the nature of stigmatising beliefs about depression and help-seeking: Implications for reducing stigma. BMC Public Health 2009; 9:61.

20. Portenoy RK, Dole V, Joseph H, Lowinson J, Rice C, Segal S, Richman BL. Pain management and chemical dependency. Evolving perspectives. JAMA 1997; 278:592-593.

21. Scimeca MM, Savage SR, Portenoy R, Lowinson J. Treatment of pain in methadone-maintained patients. Mt Sinai J Med 2000; 67:412-422.

22. Blinderman CD, Sekine R, Zhang B, Nillson M, Shaiova L. Methadone as an analgesic for patients with chronic pain in methadone maintenance treatment programs (MMTPs). J Opioid Manag 2009; 5:107-114.

23. Wessley $\mathrm{S}$. What do you think is a nondisease? BR Med / 2002; 334:912.

24. Quintner JL. The Australian RSI debate: Stereotyping and medicine. Disabil Rehabil 1995; 17:256-262.

25. Harding G, Parsons S, Rahman A, Underwood $M$. It struck me that they didn't understand pain: The specialist pain clinic experience of patients with chronic musculoskeletal pain. Arthritis Rheum (Arthritis Care Res) 2005; 53:691-696.

26. Korula M. Psycosocial aspects of pain management. Indian Journal of Anaesthesia 2008; 52:777-787. 
